\title{
Thoracic Outlet Syndrome
}

National Institute of Neurological Disorders and Stroke (NINDS)

\section{Source}

National Institute of Neurological Disorders and Stroke (NINDS). Thoracic Outlet

Syndrome Information Page.

TOS is an umbrella term that encompasses three related syndromes that involve compression of the nerves, arteries, and veins in the lower neck and upper chest area and cause pain in the arm, shoulder, and neck. Most doctors agree that TOS is caused by compression of the brachial plexus or subclavian vessels as they pass through narrow passageways leading from the base of the neck to the armpit and arm, but there is considerable disagreement about its diagnosis and treatment.

Making the diagnosis of TOS even more difficult is that a number of disorders feature symptoms similar to those of TOS, including rotator cuff injuries, cervical disc disorders, fibromyalgia, multiple sclerosis, complex regional pain syndrome, and tumors of the syrinx or spinal cord. The disorder can sometimes be diagnosed in a physical exam by tenderness in the supraclavicular area, weakness and/or a "pins and needles" feeling when elevating the hands, weakness in the fifth ("little") finger, and paleness in the palm of one or both hands when the individual raises them above the shoulders, with the fingers pointing to the ceiling. Symptoms of TOS vary depending on the type.

- Neurog enic TOS has a characteristic sign, called the Gilliatt-Sumner hand, in which there is severe wasting in the fleshy base of the thumb. Other symptoms include paresthesias (pins and needles sensation or numbness) in the fingers and hand, change in hand color, hand coldness, or dull aching pain in the neck, shoulder, and armpit.

- Venous TOS features pallor, a weak or absent pulse in the affected arm, which also may be cool to the touch and appear paler than the unaffected arm. Symptoms may include numbness, ting ling, aching, swelling of the extremity and fingers, and weakness of the neck or arm.

- Arterial TOS most prominently features change in color and cold sensitivity in the hands and fingers, swelling, heaviness, paresthesias and poor blood circulation in the arms, hands, and fingers. 
There are many causes of TOS, including physical trauma, anatomical defects, tumors that press on nerves, poor posture that causes nerve compression, pregnancy, and repetitive arm and shoulder movements and activity, such as from playing certain sports. TOS is more common in women. The onset of symptoms usually occurs between 20 and 50 years of age. Doctors usually recommend nerve conduction studies, electromyography, or imaging studies to confirm or rule out a diagnosis of TOS. 